\title{
A NOTE ON QUENCHED MODERATE DEVIATIONS FOR SINAI'S RANDOM WALK IN RANDOM ENVIRONMENT
}

\author{
Francis Comets ${ }^{1}$ And Serguei Popov ${ }^{2}$
}

\begin{abstract}
We consider the continuous time, one-dimensional random walk in random environment in Sinai's regime. We show that the probability for the particle to be, at time $t$ and in a typical environment, at a distance larger than $t^{a}(0<a<1)$ from its initial position, is $\exp \left\{-\right.$ Const $\cdot t^{a} /[(1-$ a) $\ln t](1+o(1))\}$.
\end{abstract}

Mathematics Subject Classification. 60F10, 60K37.

Received May 23, 2003. Revised October 13, 2003.

\section{INTRODUCTION AND THE RESULT}

Suppose that for all $x \in \mathbb{Z}$ we are given two positive numbers, $\omega_{x}^{+}, \omega_{x}^{-}$. Consider the continuous-time Markov chain $\xi=\left(\xi_{t}\right)_{t \geq 0}$ on $\mathbb{Z}$, which jumps from $y$ to $y+1$ with rate $\omega_{y}^{+}$, and to $y-1$ with rate $\omega_{y}^{-}$. We assume that $\omega=\left(\omega_{x}^{+}, \omega_{x}^{-}\right)_{x \in \mathbb{Z}}$ is a fixed realization of an i.i.d. sequence of positive random vectors. We refer to $\omega$ as the environment, and to $\xi$ as the random walk in the random environment $\omega$. We will denote by $\mathbb{P}, \mathbb{E}$ the probability and expectation with respect to $\omega$, and by $\mathrm{P}_{\omega}^{x}, \mathrm{E}_{\omega}^{x}$ the (so-called "quenched") probability and expectation for random walks starting from $x$ in the fixed environment $\omega$. In this paper we study only the case of Sinai's regime, which means that the following condition is satisfied:

Condition S. We have

$$
\mathbb{E} \ln \frac{\omega_{0}^{+}}{\omega_{0}^{-}}=0, \quad \sigma^{2}:=\mathbb{E} \ln ^{2} \frac{\omega_{0}^{+}}{\omega_{0}^{-}} \in(0,+\infty) .
$$

For technical reasons we also need the following uniform ellipticity condition:

Condition B. There exists a positive number $C$ such that

$$
C^{-1} \leq \omega_{0}^{+} \leq C, \quad C^{-1} \leq \omega_{0}^{-} \leq C \quad \mathbb{P} \text {-a.s. }
$$

Keywords and phrases. Random walk in random environment, Sinai's regime, $t$-stable point, moderate deviations.

1 Université Paris 7, UFR de Mathématiques, case 7012, 2, place Jussieu, 75251 Paris Cedex 05, France;

e-mail: comets@math.jussieu.fr

Partially supported by CNRS (UMR 7599 "Probabilités et Modèles Aléatoires") and by the "Réseau Mathématiques FranceBrésil".

2 Instituto de Matemática e Estatística, Universidade de São Paulo, Rua do Matão 1010, CEP 05508-090, São Paulo SP, Brasil; e-mail: popov@ime.usp.br

Partially supported by CNPq (302981/02-0), and by the "Rede Matemática Brasil-França". 
This model has been much studied in last 30 years in its discrete version, and the state of art is given in the recent surveys $[5,6,9]$ with different points of view. The discrete time random walk in random environment is the Markov chain embedded in the present continuous time process. A striking feature of this regime is the ultraslow diffusion of the particle, due to the existence of traps in typical environments: a celebrated result of Sinai [8] states that $\xi_{t}$ is of order $\ln ^{2} t$ for large $t$, more precisely, that the ratio converges in law to a non-degenerate random variable. One naturally expects that the deviation properties of the walk reflect this slowdown. At the level of large deviations in the discrete case [2], it holds that $\mathrm{P}_{\omega}^{0}\left[\xi_{t} \geq v t\right]=\exp \{-t(I(v)+o(1))\},(v \geq 0$, $t \rightarrow \infty)$ with a strictly positive rate function $I$ for $v>0$, and only the (conjectured) non-analyticity of $I$ at 0 can support the above expectation. Theorem 2.3 of [1] reveals a non-standard behavior for smaller deviations, precisely

$$
\mathrm{P}_{\omega}^{0}\left[\xi_{t} \geq z\right]=\exp \left\{-\sigma^{2} z /(2 \ln t)(1+o(1))\right\}
$$

for $\ln ^{2} t \ln \ln \ln t \ll z \leq \ln ^{M} t(M>2)$ as $t \rightarrow \infty$. (The expression $f(t) \ll g(t)$ means that $f(t)=o(g(t))$ as $t \rightarrow$ $\infty$.) This estimate supports the above expectation, since the rate of decay is much larger than the standard rate $z^{2} / t$ of decay of moderate deviations (e.g., in the Gaussian case). The restriction on small values of $z$ cannot be removed, as shown by an earlier result of $\mathrm{Hu}$ and Shi [3] in the discrete case, $\lim \sup \xi_{t} /\left(\ln ^{2} t \ln \ln \ln t\right)=8 /\left(\pi^{2} \sigma^{2}\right)$. In fact, for $z=a \ln ^{2} t$ with $a>0$ fixed, it follows from Theorem 2.1 in [1] that $\mathrm{P}_{\omega}^{0}\left[\xi_{t} \geq a \ln ^{2} t\right]=t^{-\beta_{t}+o(1)}$, where the random variable $\beta_{t}=\beta_{t}(a)$ converges in law to a non-negative random limit (which has a strictly positive mass at 0 ). The purpose of this short note is to extend our previous result (1) for moderate deviation to larger values of $z$.

Fix a positive function $\varphi(t)$ such that $\varphi(t) \rightarrow \infty$ as $t \rightarrow \infty$, and a positive number $a<1$. For each $t>\mathrm{e}^{\mathrm{e}}$, define an interval

$$
R_{t}(\varphi, a)=\left[\ln ^{2} t \times \ln \ln \ln t \times \varphi(t), t^{a}\right] .
$$

Our result in this paper is a refinement of $(1)$ :

Theorem 1.1. Let $\xi_{t}$ be the random walk in random environment satisfying Conditions $S$ and $B$. For any a and $\varphi$ as above, we have

$$
\sup _{z \in R_{t}(\varphi, a)}\left|\frac{2(\ln t-\ln z) \ln \mathrm{P}_{\omega}^{0}\left[\xi_{t} \geq z\right]}{\sigma^{2} z}+1\right| \longrightarrow 0 \quad \mathbb{P} \text {-a.s. }
$$

as $t \rightarrow \infty$. The same result holds if one substitutes $\xi_{t}$ by $\max _{s \leq t} \xi_{s}$.

Remark 1.1. (i) Following [1], we have chosen the continuous time, but, just as in [1], the result remains valid in the discrete-time case as well.

(ii) While finishing this note, we received the preprint [4] where $\mathrm{Hu}$ and Shi obtain the moderate deviation principle for the one-dimensional diffusion in a Brownian potential, which is the continuous-space analogue of the random walk in random environment. Their approach is based on fine tools from stochastic calculus, and they cover both the quenched and the annealed case. Their quenched result, which is in agreement with our Theorem 1.1, is obtained by studying the Laplace transform of hitting times via Kotani's lemma.

(iii) The present approach is a direct, pathwise approach. From the proof below, we can understand how the walk behaves, when performing such a rare event. In particular, consider the sequence of traps between sites 0 and $z$, with depth larger than the "critical depth" $\ln (t / z)$. Typically for a rare event, the walk shares equally (at least on a logarithmic scale) the total time $t$ in the various traps with such depths (see e.g. (18) below).

(iv) One may be interested about what happens in the situation when $z=o(t)$, but $\ln z / \ln t \rightarrow 1$, i.e., how to refine Theorem 1.1 in order to remove the fixed constant $a<1$. We can conjecture (by analogy with [4]) that Theorem 1.1 remains true when $\ln \ln t=o(\ln (t / z))$. It is our hope that the method of the present paper could be refined to be able to deal with this situation; unfortunately, at the present time it is unclear to us how to do that. 


\section{Preliminaries}

In this section we introduce more notations and establish (or recall) some properties needed in the course of the proof of Theorem 1.1 .

Given the realization of the random environment $\omega$, define for $x \in \mathbb{Z}$

$$
V(x)=\left\{\begin{array}{cc}
\sum_{i=0}^{x-1} \ln \frac{\omega_{i}^{-}}{\omega_{i}^{+}}, & x>0, \\
0, & x=0 \\
\sum_{i=x+1}^{0} \ln \frac{\omega_{i}^{+}}{\omega_{i}^{-}}, & x<0 .
\end{array}\right.
$$

The notion of $t$-stable point is essential in this paper.

Definition 2.1. Let $t>1$ and, for $m \in \mathbb{Z}$, denote by $l=l(t, m)$ the largest $x<m$ such that $V(x) \geq V(m)+\ln t$ (with the convention $l=-\infty$ if no such $x$ exists), and by $r$ the smallest $x>m$ such that $V(x) \geq V(m)+\ln t$ (with the similar convention).

We say that $m$ is a $t$-stable point if $m$ is the smallest point on the interval $[l, r]$ which satisfies $V(m)=$ $\min _{x \in[l, r]} V(x)$.

We alert the reader that in [1] a slightly different approach was used. There, the potential $V$ was coupled, from the very beginning, with a two-sided Brownian motion $W$ by using the KMT strong approximation theorem, and $t$-stable points were defined in terms of $W$. In the present paper we cannot use this approach because the interval where the coupling would take place is too large; instead, we define the $t$-stable points in terms of the "true" potential $V$. This causes some additional complications because of the lack of the scaling properties (see Lems. 2.1-2.3 below).

Since $\limsup _{x \rightarrow \pm \infty} V(x)=-\liminf _{x \rightarrow \pm \infty} V(x)=+\infty$, the set of $t$-stable points is infinite $\mathbb{P}$-a.s., as well as its intersections with $(0,+\infty)$ and $(-\infty, 0)$. Let

$$
\mathcal{S}_{t}=\left\{\ldots, m_{t}^{-1}, m_{t}^{0}, m_{t}^{1}, \ldots\right\} \subset \mathbb{Z},
$$

where $m_{t}^{i}<m_{t}^{i+1}$ for all $i \in \mathbb{Z}$, be the set of all $t$-stable points labeled in such a way that $m_{t}^{0}<0 \leq m_{t}^{1}$. Also, let $h_{t}^{i}, i \in \mathbb{Z}$, be the smallest point such that

$$
V\left(h_{t}^{i}\right)=\max _{x \in\left[m_{t}^{i}, m_{t}^{i+1}\right]} V(x)
$$

We will refer to the interval $\left[h_{t}^{i-1}, h_{t}^{i}\right]$ as the $t$-stable well of $m_{t}^{i}$, and to the points $h_{t}^{i}$ as passes. For $t>1$ and $i \in \mathbb{Z}$ define the non-negative random variables (see Fig. 1)

$$
\begin{aligned}
\zeta_{i}(t) & =m_{t}^{i+1}-m_{t}^{i}, \\
\eta_{i}(t) & =V\left(h_{t}^{i}\right)-V\left(m_{t}^{i}\right)-\ln t .
\end{aligned}
$$

It is not difficult to see that the random variables $\eta_{i}(t), i=1,2,3, \ldots$, are i.i.d.; moreover, the same holds for $\zeta_{i}(t), i=1,2,3, \ldots$ The last claim follows from the following fact: if for some $C_{0}>0$ we define $\tau_{1}=\min \{x>$ $\left.0: V(x)-\min _{0 \leq y \leq x} V(y)>C_{0}\right\}$ and $\tau_{2}=\min \left\{x>0: V(x)=\min _{0 \leq y \leq \tau_{1}} V(y)\right\}$, then $\tau_{2}$ is independent from $\tau_{1}-\tau_{2}$ and $V$ starts afresh from the stopping time $\tau_{1}$. We need also to define the rescaled variables

$$
\tilde{\zeta}_{i}(s):=s^{-2} \zeta_{i}\left(\mathrm{e}^{s}\right), \quad \tilde{\eta}_{i}(s):=s^{-1} \eta_{i}\left(\mathrm{e}^{s}\right)
$$

As $s \rightarrow \infty$, it is natural to approximate the distribution of $\tilde{\zeta}_{i}(s)$ and $\tilde{\eta}_{i}(s)$ by the distribution of the random variables $\hat{\zeta}, \hat{\eta}$, defined as follows. 


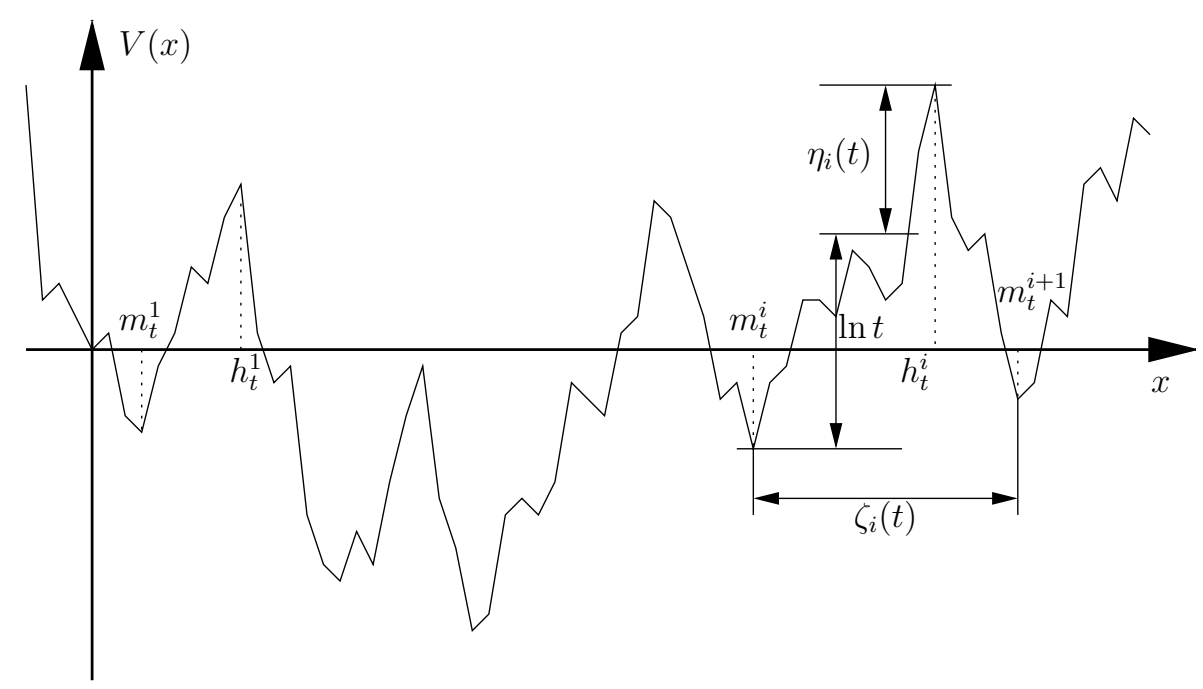

FIGURE 1. On the definition of $m_{t}^{i}, h_{t}^{i}, \zeta_{i}(t), \eta_{i}(t)$.

Let $W(x), x \in \mathbb{R}$, be the two-sided Brownian motion with diffusion constant $\sigma$, and let us repeat the above constructions with $W$ instead of $V$. Let $\left\{\ldots, \hat{m}_{t}^{-1}, \hat{m}_{t}^{0}, \hat{m}_{t}^{1}, \ldots\right\}$, be the set of $t$-stable points for $W$, i.e., defined as in Definition 2.1 but with $W$ instead of $V$ (and labeled in such a way that $\hat{m}_{t}^{0}<0 \leq \hat{m}_{t}^{1}, \hat{m}_{t}^{i}<\hat{m}_{t}^{i+1}$ for all $i \in \mathbb{Z})$, and let $\left(\hat{h}_{t}^{i}, i \in \mathbb{Z}\right)$ be the corresponding passes. Then, we define $\hat{\zeta}=\hat{m}_{e}^{2}-\hat{m}_{e}^{1}$, and $\hat{\eta}=W\left(\hat{h}_{e}^{1}\right)-W\left(\hat{m}_{e}^{1}\right)-1$. We emphasize that the following estimates, as well as those in Lemmas 2.3 and 2.5, are uniform.

Lemma 2.1. There exist positive constants $K_{i}, i=1, \ldots, 6$, such that for for all s large enough and all $n$

$$
\begin{aligned}
\mathbb{P}\left[\tilde{\zeta}_{1}(s)>n\right] & \leq K_{1} \mathrm{e}^{-K_{2} n}, \\
\mathbb{P}\left[\tilde{\eta}_{1}(s)>n\right] & \leq K_{3} \mathrm{e}^{-K_{4} n}, \\
\mathbb{P}[\hat{\zeta}>n] & \leq K_{5} \mathrm{e}^{-K_{6} n}, \\
\mathbb{P}[\hat{\eta}>n] & =\mathrm{e}^{-n} .
\end{aligned}
$$

Proof. We begin by proving (6). By Condition B, there exists a constant $D_{0} \in(0 ;+\infty)$ such that $\mid V(x)-$ $V(x+1) \mid<D_{0}, \mathbb{P}$-a.s. Suppose in the sequel that $s>2 D_{0}$ and define

$$
\tilde{\tau}=\min \left\{x>0: V(x) \notin\left[-s ; s-D_{0}\right]\right\}
$$

Define also $A_{s}=\left\{V(\tilde{\tau})>s-D_{0}\right\}$ and $\tilde{\tau}_{1}=\min \left\{x>m_{\mathrm{e}^{s}}^{1}: V(x)-V\left(m_{\mathrm{e}^{s}}^{1}\right) \geq s\right\}$. The event $\left\{\eta_{1}\left(\mathrm{e}^{s}\right)>s\right\}$ contains the event

$$
\left\{\min \left\{x>\tilde{\tau}_{1}: V(x)-V\left(\tilde{\tau}_{1}\right)>s-D_{0}\right\}<\min \left\{x>\tilde{\tau}_{1}: V(x)-V\left(\tilde{\tau}_{1}\right)<-s\right\}\right\}
$$

which has the same probability as $A_{s}$. Iterating this argument, and since that $\tilde{\tau}_{1}$ is a stopping time and $V$ has independent increments, we can write

$$
\mathbb{P}\left[\tilde{\eta}_{1}(s)>n\right]=\mathbb{P}\left[\eta_{1}\left(\mathrm{e}^{s}\right)>s n\right] \leq\left(\mathbb{P}\left[A_{s}\right]\right)^{n}
$$


Since $V(\cdot \wedge \tilde{\tau})$ is a bounded martingale, we have

$$
\begin{aligned}
0=\mathbb{E} V(\tilde{\tau}) & \geq\left(s-D_{0}\right) \mathbb{P}\left[A_{s}\right]-\left(s+D_{0}\right)\left(1-\mathbb{P}\left[A_{s}\right]\right) \\
& =2 s \mathbb{P}\left[A_{s}\right]-\left(s+D_{0}\right),
\end{aligned}
$$

so $\mathbb{P}\left[A_{s}\right] \leq \frac{1}{2}\left(1+D_{0} / s\right)$, and we get (6) from (10).

Now, we prove (5). Define

$$
B_{s}=\left\{V\left(s^{2} / 4\right)-V(0)<-s, V\left(s^{2} / 2\right)-V\left(s^{2} / 4\right)>s, V\left(3 s^{2} / 4\right)-V\left(s^{2} / 2\right)<-s, V\left(s^{2}\right)-V\left(3 s^{2} / 4\right)>s\right\} .
$$

Clearly, on $B_{s}$ there are at least two $e^{s}$-stable points in the interval $\left[0, s^{2}\right]$. Iterating this argument as in (10), we can write

$$
\mathbb{P}\left[\tilde{\zeta}_{1}(s)>n\right]=\mathbb{P}\left[\zeta_{1}\left(e^{s}\right)>s^{2} n\right] \leq\left(1-\mathbb{P}\left[B_{s}\right]\right)^{n} .
$$

By Central Limit Theorem, there exist $s_{0}, D_{1}>0$ such that $\mathbb{P}\left[B_{s}\right]>D_{1}$ for all $s \geq s_{0}$, and we get (5) from (11).

The proof of (7) proceeds quite analogously to the proof of (5), and (8) is obvious, because (see e.g. Sect. 6 of [1]) $\hat{\eta}$ has exponential distribution with parameter 1.

Lemma 2.2. As $s \rightarrow \infty$, we have the following convergence in law:

$$
\begin{aligned}
& \tilde{\zeta}_{1}(s) \longrightarrow \hat{\zeta}, \\
& \tilde{\eta}_{1}(s) \longrightarrow \hat{\eta} .
\end{aligned}
$$

Proof. This is an elementary consequence of Donsker's invariance principle.

Lemma 2.3. For any $b_{1}>2 \sigma^{-2}$ and any $b_{2}>1$ there exist positive constants $K_{7,8}=K_{7,8}\left(b_{1}\right)$ and $K_{9,10}=$ $K_{9,10}\left(b_{2}\right)$ such that for all s large enough and for all $n$

$$
\begin{aligned}
\mathbb{P}\left[\frac{1}{n} \sum_{j=1}^{n} \tilde{\zeta}_{j}(s)>b_{1}\right] & \leq K_{7} \mathrm{e}^{-K_{8} n}, \\
\mathbb{P}\left[\frac{1}{n} \sum_{j=1}^{n} \tilde{\eta}_{j}(s)>b_{2}\right] & \leq K_{9} \mathrm{e}^{-K_{10} n} .
\end{aligned}
$$

The same estimates are valid if we replace the inequalities " $>$ " by " $<$ " in (14) and (15) and suppose that $b_{1}<2 \sigma^{-2}, b_{2}<1$.

Proof. First, note that $\mathbb{E} \hat{\zeta}=2 \sigma^{-2}$ and $\mathbb{E} \hat{\eta}=1$ ( $c f$. Sect. 6 of [1]). Then, by Chernoff's theorem we get that for all $n, s$ and for any $\lambda_{0}>0$

$$
\mathbb{P}\left[\frac{1}{n} \sum_{j=1}^{n} \tilde{\zeta}_{j}(s)>b_{1}\right] \leq \exp \left\{-n \psi_{s, \lambda_{0}}\left(b_{1}\right)\right\}
$$

where

$$
\psi_{s, \lambda_{0}}(b)=\sup _{\lambda \in\left(0, \lambda_{0}\right]}\left(\lambda b-\ln \mathbb{E} \mathrm{e}^{\lambda \tilde{\zeta}_{1}(s)}\right)
$$

Since $b_{1}>\mathbb{E} \hat{\zeta}$, the function

$$
\psi_{\lambda_{0}}(b)=\sup _{\lambda \in\left(0, \lambda_{0}\right]}\left(\lambda b-\ln \mathbb{E} \mathrm{e}^{\lambda \hat{\zeta}}\right)
$$

satisfies $\psi_{\lambda_{0}}\left(b_{1}\right)>0$, and, from Lemmas 2.1 and 2.2 one gets that (at least for small enough $\left.\lambda\right) \mathbb{E} \mathrm{e}^{\lambda \tilde{\zeta}_{1}(s)} \rightarrow \mathbb{E} \mathrm{e}^{\lambda \hat{\zeta}}$ as $s \rightarrow \infty$. This implies that, for small enough $\lambda_{0}, \psi_{s, \lambda_{0}}\left(b_{1}\right) \rightarrow \psi_{\lambda_{0}}\left(b_{1}\right)>0$ as $s \rightarrow \infty$, thus proving (14). The proof of the other claims of the lemma proceeds in the same way. 
Now, we need to recall a fact from [1] which concerns the cost of escaping from $t$-stable wells. Let $m<m^{\prime}$ be two neighboring $t$-stable points and $h$ be the highest pass between them. By $\tau_{x}$ we denote the first moment when the random walk $\xi$ hits the point $x$. The following two results are stated without proof.

Lemma 2.4 (Lem. 3.4 of [1]). There is a constant $K_{11}>0$ such that for any $x>h$ and any $s$ we have

$$
\mathrm{P}_{\omega}^{m}\left[\tau_{x}<s\right] \leq K_{11} s \mathrm{e}^{-V(h)+V(m)}
$$

Finally, we recall Chernoff's bound for the binomial distribution:

Lemma 2.5 (see e.g. [7], p. 68). Let $\left\{X_{i}, i \geq 1\right\}$ be i.i.d. random variables with $\mathbb{P}\left[X_{i}=1\right]=p$ and $\mathbb{P}\left[X_{i}=0\right]=$ $1-p$. Then for any $0<p<a<1$ and for any $n \geq 1$ we have

$$
\mathbb{P}\left[\frac{1}{n} \sum_{i=1}^{n} X_{i} \geq a\right] \leq \exp \{-n H(a, p)\}
$$

where

$$
H(a, p)=a \log \frac{a}{p}+(1-a) \log \frac{1-a}{1-p}>0 .
$$

If $0<a<p<1$, then (16) holds with $\mathbb{P}\left[n^{-1} \sum_{i=1}^{n} X_{i} \leq a\right]$ in the left-hand side.

\section{Proof of Theorem 1.1}

\section{Upper bound for $\mathrm{P}_{\omega}^{0}\left[\max _{s \leq t} \xi_{s} \geq z\right]$}

For any $z \in R_{t}(\varphi, a)$ denote

$$
\gamma_{t}^{z}=\frac{\ln z}{\ln t}
$$

so that $t^{1-\gamma_{t}^{z}}=t / z$. For any $t>1$ define $N_{\omega}(t, z)$ by

$$
N_{\omega}(t, z)=i \quad \text { if } \quad h_{t}^{i} \leq z<h_{t}^{i+1}
$$

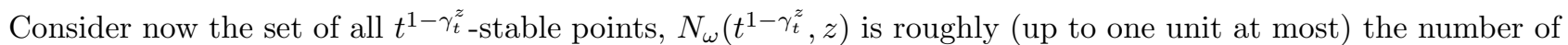
them between 0 and $z$. Abbreviating $m^{i}:=m_{t^{1-\gamma_{t}^{z}}}^{i}, h^{i}:=h_{t^{1-\gamma_{t}^{z}}}^{i}$, define also $\kappa_{i}=\tau_{h^{i}}-\tau_{m^{i}}$. The quantity $\kappa_{i}$ is the time, after reaching the $i$ th $t^{1-\gamma_{t}^{z}}$-stable point, to climb the wall separating it from the $(i+1)$ th one. Write

$$
\begin{aligned}
\mathrm{P}_{\omega}^{0}\left[\max _{s \leq t} \xi_{s} \geq z\right] & =\mathrm{P}_{\omega}^{0}\left[\tau_{z} \leq t\right] \\
& \leq \mathrm{P}_{\omega}^{0}\left[\sum_{i=1}^{N_{\omega}\left(t^{1-\gamma_{t}^{z}}, z\right)} \kappa_{i} \leq t\right]
\end{aligned}
$$

For $i=1, \ldots N_{\omega}\left(t^{1-\gamma_{t}^{z}}, z\right)$, let $Y_{i}$ be the indicator function

$$
Y_{i}=\mathbf{1}\left\{\kappa_{i} \leq \frac{t \ln t}{N_{\omega}\left(t^{1-\gamma_{t}^{z}}, z\right)}\right\}
$$

Fix some $h>0$ and define

$$
J_{k}^{(h)}(t, z)=\left\{1 \leq i \leq N_{\omega}\left(t^{1-\gamma_{t}^{z}}, z\right): \tilde{\eta}_{i}(\ln t-\ln z) \in[k h,(k+1) h)\right\},
$$


with $\tilde{\eta}_{i}$ given in (4), and

$$
\theta_{k}^{(h)}=\sup _{i \in J_{k}^{(h)}(t, z)} \mathrm{P}_{\omega}^{0}\left[Y_{i}=1\right]=\sup _{i \in J_{k}^{(h)}(t, z)} \mathrm{P}_{\omega}^{m^{i}}\left[\tau_{h^{i}} \leq \frac{t \ln t}{N_{\omega}\left(t^{1-\gamma_{t}^{z}}, z\right)}\right],
$$

$k=0,1,2, \ldots$, with the convention that the supremum over an empty set is 1 . Now, from (17) and by the Markov property of the walk, we obtain for any fixed positive integer $M$

$$
\begin{aligned}
\mathrm{P}_{\omega}^{0}\left[\max _{s \leq t} \xi_{s} \geq z\right] & \leq \mathrm{P}_{\omega}^{0}\left[\sum_{i=1}^{N_{\omega}\left(t^{1-\gamma_{t}^{z}}, z\right)}\left(1-Y_{i}\right) \kappa_{i} \leq t\right] \\
& \leq \mathrm{P}_{\omega}^{0}\left[\sum_{i=1}^{N_{\omega}\left(t^{1-\gamma_{t}^{z}}, z\right)} Y_{i} \geq\left(1-\ln ^{-1} t\right) N_{\omega}\left(t^{1-\gamma_{t}^{z}}, z\right)\right] \\
& \leq \prod_{k=0}^{M} \mathrm{P}_{\omega}^{0}\left[\sum_{i \in J_{k}^{(h)}(t, z)} Y_{i} \geq\left|J_{k}^{(h)}(t, z)\right|\left(1-\frac{N_{\omega}\left(t^{1-\gamma_{t}^{z}}, z\right)}{\left|J_{k}^{(h)}(t, z)\right| \ln t}\right)\right] \\
& \leq \exp \left\{-\sum_{k=0}^{M}\left|J_{k}^{(h)}(t, z)\right| H\left(1-\frac{N_{\omega}\left(t^{1-\gamma_{t}^{z}}, z\right)}{\left|J_{k}^{(h)}(t, z)\right| \ln t}, \theta_{k}^{(h)}\right)\right\},
\end{aligned}
$$

using also Lemma 2.5 and the stochastic monotonicity of Bernoulli variables with respect to their parameter to get the last line. Let us fix $\delta>0$ and divide the time interval $[\mathrm{e},+\infty)$ into a countable collection of intervals $I_{n}:=\left[\mathrm{e}^{(1+\delta)^{n}}, \mathrm{e}^{(1+\delta)^{n+1}}\right), n=0,1,2, \ldots$ Define also

$$
\begin{aligned}
r^{(n)} & =\inf \bigcup_{t \in I_{n}} R_{t}(\varphi, a)=(1+\delta)^{2 n} \varphi\left(\mathrm{e}^{(1+\delta)^{n}}\right) \ln (n \ln (1+\delta)), \\
\mathcal{R}_{n} & =\left\{(t, z): z \in R_{t}(\varphi, a), t \in I_{n}\right\},
\end{aligned}
$$

and, for some fixed $\varepsilon^{\prime}>0$ and $m=0, \ldots,\left\lceil a / \varepsilon^{\prime}\right\rceil$

$$
\Gamma_{n, m}^{\varepsilon^{\prime}}=\left\{(t, z) \in \mathcal{R}_{n}: \gamma_{t}^{z} \in\left(m \varepsilon^{\prime},(m+1) \varepsilon^{\prime}\right]\right\} .
$$

Fix $\varepsilon>0$ and consider the events

$$
\begin{aligned}
B_{n}^{\varepsilon} & =\left\{\left|\frac{2 N_{\omega}\left(t^{1-\gamma_{t}^{z}}, z\right)(\ln t-\ln z)^{2}}{\sigma^{2} z}-1\right|<\varepsilon \text { for all }(t, z) \in \mathcal{R}_{n}\right\}, \\
D_{n}^{\varepsilon, k, h} & =\left\{\left|\frac{2\left|J_{k}^{(h)}(t, z)\right|(\ln t-\ln z)^{2}}{\sigma^{2} z \mathbb{P}[k h \leq \hat{\eta}<(k+1) h]}-1\right|<\varepsilon \text { for all }(t, z) \in \mathcal{R}_{n}\right\} .
\end{aligned}
$$

We need to bound the probabilities of these events from below. Write

$$
\begin{aligned}
\mathbb{P}\left[\left(B_{n}^{\varepsilon}\right)^{c}\right] \leq & \mathbb{P}\left[N_{\omega}\left(t^{1-\gamma_{t}^{z}}, z\right)>\frac{(1+\varepsilon) \sigma^{2} z}{2\left(1-\gamma_{t}^{z}\right) \ln ^{2} t} \text { for some }(t, z) \in \mathcal{R}_{n}\right] \\
& +\mathbb{P}\left[N_{\omega}\left(t^{1-\gamma_{t}^{z}}, z\right)<\frac{(1-\varepsilon) \sigma^{2} z}{2\left(1-\gamma_{t}^{z}\right) \ln ^{2} t} \text { for some }(t, z) \in \mathcal{R}_{n}\right] \\
=: & T_{1}+T_{2} .
\end{aligned}
$$


First, consider the term $T_{1}$. Abbreviate $g_{0}=g_{0}(m)=\frac{1}{2}(1+\varepsilon)\left(1-m \varepsilon^{\prime}\right)^{-2}(1+\delta)^{-2(n+1)} \sigma^{2} z$, and $f_{0}=$ $f_{0}(m)=\left(1-(m+1) \varepsilon^{\prime}\right)(1+\delta)^{n}$. Since $\mathcal{S}_{t} \subset \mathcal{S}_{t^{\prime}}$ for $t^{\prime} \leq t$, we have

$$
\begin{aligned}
& T_{1} \leq \sum_{m=0}^{\left\lceil a / \varepsilon^{\prime}\right\rceil} \mathbb{P}\left[N_{\omega}\left(t^{1-\gamma_{t}^{z}}, z\right)>(1+\varepsilon) \frac{\sigma^{2} z}{2\left(1-\gamma_{t}^{z}\right) \ln ^{2} t} \text { for some }(t, z) \in \Gamma_{n, m}^{\varepsilon^{\prime}}\right] \\
& \leq \sum_{m=0}^{\left\lceil a / \varepsilon^{\prime}\right\rceil} \mathbb{P}\left[N_{\omega}\left(\mathrm{e}^{f_{0}}, z\right)>g_{0} \text { for some } z \geq r^{(n)}\right] \\
& \leq \sum_{m=0}^{\left\lceil a / \varepsilon^{\prime}\right\rceil} \mathbb{P}\left[\sum_{i=1}^{g_{0}} \zeta_{i}\left(\mathrm{e}^{f_{0}}\right)<z \text { for some } z \geq r^{(n)}\right] \\
&=\sum_{m=0}^{\left\lceil a / \varepsilon^{\prime}\right\rceil} \mathbb{P}\left[\sum_{i=1}^{g_{0}} \tilde{\zeta}_{i}\left(f_{0}\right)<f_{0}^{-2} z \text { for some } z \geq r^{(n)}\right] \\
& \leq \sum_{m=0}^{\left\lceil a / \varepsilon^{\prime}\right\rceil} \mathbb{P}\left[\frac{1}{g_{0}} \sum_{i=1}^{g_{0}} \tilde{\zeta}_{i}\left(f_{0}\right)<\frac{2}{\sigma^{2}} \Psi\left(\varepsilon, \varepsilon^{\prime}, \delta\right)\right. \\
&\left.\quad \text { for some } g_{0} \geq \frac{(1+\varepsilon) \sigma^{2}}{2\left(1-m \varepsilon^{\prime}\right)^{2}\left(1+\delta^{2}\right)} \varphi\left(\mathrm{e}^{(1+\delta)^{n}}\right) \ln (n \ln (1+\delta))\right]
\end{aligned}
$$

where $\Psi\left(\varepsilon, \varepsilon^{\prime}, \delta\right)=(1+\varepsilon)^{-1}(1+\delta)^{2}\left(1+\varepsilon^{\prime} / a\right)^{2}$. Clearly, a similar estimate is available for the term $T_{2}$ as well. Now, for any fixed $\varepsilon$ one can always choose small enough $\delta, \varepsilon^{\prime}$ in such a way that $\Psi\left(\varepsilon, \varepsilon^{\prime}, \delta\right)<1$. So, from Lemma 2.3 we obtain that for some positive numbers $K_{12}, K_{13}$ (which do not depend on $n$ )

$$
\mathbb{P}\left[B_{n}^{\varepsilon}\right] \geq 1-K_{12} \exp \left\{-K_{13} \varphi\left(\mathrm{e}^{(1+\delta)^{n}}\right) \ln n\right\} .
$$

On the other hand, by Lemma 2.2 , it holds that for any $i \geq 1$

$$
\mathbb{P}\left[i \in J_{k}^{(h)}(t, z)\right] \rightarrow \mathbb{P}[k h \leq \hat{\eta}<(k+1) h]
$$

as $t \rightarrow \infty$, uniformly in $z \in R_{t}(\varphi, a)$. Similar to the derivation of (20), an elementary computation shows that for some positive numbers $K_{14}, K_{15}$ not depending on $n$

$$
\mathbb{P}\left[D_{n}^{\varepsilon, k, h}\right] \geq 1-K_{14} \exp \left\{-K_{15} \varphi\left(\mathrm{e}^{(1+\delta)^{n}}\right) \ln n\right\} .
$$

Taking into account that $\varphi \rightarrow \infty,(20)$ and (21) imply, via the Borel-Cantelli lemma, that for any fixed $\varepsilon, k, h$ all but a finite number of events $B_{n}^{\varepsilon}, D_{n}^{\varepsilon, k, h}$ occur. Note also that for any $\varepsilon^{\prime \prime}>0$, by Lemma 2.4 it holds that $\theta_{k}^{(h)} \leq t^{-k h\left(1-\gamma_{t}^{z}\right)\left(1-\varepsilon^{\prime \prime}\right)}$ for all $t$ large enough. Then, we obtain from (19) that on $B_{n}^{\varepsilon} \cap D_{n}^{\varepsilon, k, h}$

$$
\begin{aligned}
\ln \mathrm{P}_{\omega}^{0}\left[\max _{s \leq t} \xi_{s} \geq z\right] \leq & -(1-\varepsilon) \frac{\sigma^{2} z}{2(\ln t-\ln z)^{2}} \sum_{k=0}^{M} \mathbb{P}[k h \leq \hat{\eta}<(k+1) h] \\
& \times H\left(1-\frac{(1+\varepsilon) \mathbb{P}^{-1}[k h \leq \hat{\eta}<(k+1) h]}{(1-\varepsilon) \ln t}, t^{-k h\left(1-\gamma_{t}^{z}\right)\left(1-\varepsilon^{\prime \prime}\right)}\right) .
\end{aligned}
$$

For fixed $k, h$, it holds that the first argument of the function $H(\cdot, \cdot)$ in the above display tends to 1 as $t \rightarrow \infty$, so the value of $H$ is at least $k h\left(1-\gamma_{t}^{z}\right)\left(1-2 \varepsilon^{\prime \prime}\right) \ln t$ for all $t$ large enough. By choosing $h$ small and $M$ large, 
we can make

$$
\sum_{k=0}^{M} k h \mathbb{P}[k h \leq \hat{\eta}<(k+1) h]
$$

arbitrarily close to $1=\mathbb{E} \hat{\eta}$. So, gathering the pieces, we arrive to the following result:

$$
\limsup _{t \rightarrow \infty} \sup _{z \in R_{t}(\varphi, a)} \frac{2(\ln t-\ln z) \ln \mathrm{P}_{\omega}^{0}\left[\max _{s \leq t} \xi_{s} \geq z\right]}{\sigma^{2} z} \leq-1 \quad \mathbb{P} \text {-a.s. }
$$

\section{Lower bound for $\mathrm{P}_{\omega}^{0}\left[\xi_{t} \geq z\right]$}

Contrary to the upper bound, the derivation of the lower bound requires essentially no new ideas compared to [1]. We will not repeat the full details, but in order to keep the paper self-contained, we briefly describe the main steps needed to obtain the counterpart of $(22)$.

Step 1. Consider all the $t^{1-\gamma_{t}^{z}}$-stable points between 0 and $z$. From Lemmas 2.1 and 2.2 it is elementary to get that $\mathbb{E} \tilde{\zeta}_{1}(s) \rightarrow \mathbb{E} \hat{\zeta}$ as $s \rightarrow \infty$, so the average distance between two neighboring $t^{1-\gamma_{t}^{z}}$-stable points is roughly

$$
\frac{2}{\sigma^{2}}\left(1-\gamma_{t}^{z}\right)^{2} \ln ^{2} t=\frac{2}{\sigma^{2}}(\ln t-\ln z)^{2}
$$

Hence, the number of $t^{1-\gamma_{t}^{z}}$-stable points between 0 and $z$ is approximately $\frac{\sigma^{2} z}{2(\ln t-\ln z)^{2}}$. This is, in fact, an informal description of what have been done to obtain (20).

Step 2. As in [1], we use the following strategy to go from 0 to $z$. If $N$ is the number of $t^{1-\gamma_{t}^{z}}$-stable points to pass through, we give the particle $t / N$ time units to perform the passage between each pair of two neighboring $t^{1-\gamma_{t}^{z}}$-stable points. As mentioned in the previous paragraph, $N$ can be well controlled, so, up to constant and logarithmic terms, the particle disposes of $t^{1-\gamma_{t}^{z}}$ time units to perform each passage. By the counterpart of Lemma 2.4 (which we prefer not to formulate here; see Lem. 3.6 of [1]) the probability that the particle manages to pass from $i$-th to $(i+1)$-th $t^{1-\gamma_{t}^{z}}$-stable points in $t^{1-\gamma_{t}^{z}}$ time units is (roughly) at least $\exp \left\{-\eta_{i}\left(t^{1-\gamma_{t}^{z}}\right)\right\}$. Using the fact that $\mathbb{E} \tilde{\eta}_{1}(s) \rightarrow \mathbb{E} \hat{\eta}$ as $s \rightarrow \infty$, we get that the total cost is around $\exp \left\{-\frac{\sigma^{2} z}{2(\ln t-\ln z)}\right\}$.

Step 3. Having passed through all the $t^{1-\gamma_{t}^{z}}$-stable points between 0 and $z$, the particle then finds the first $t^{1-\gamma_{t}^{z}}$-stable point to the right of $z$ (the cost of this is small compared to the cost of passing from 0 to $z$ ) which is also $t^{2}$-stable, and such that $z$ is not in its $t^{2}$-stable well. Then, the particle will spend there the rest of the time (up to time $t$ ) with a large probability. that

The above argument can be made rigorous as in [1] (proof of the lower bound of Th. 2.3, Sect. 5) to assure

$$
\liminf _{t \rightarrow \infty} \inf _{z \in R_{t}(\varphi, a)} \frac{2(\ln t-\ln z) \ln \mathrm{P}_{\omega}^{0}\left[\xi_{t} \geq z\right]}{\sigma^{2} z} \geq-1 \quad \mathbb{P} \text {-a.s. }
$$

In comparison with [1], there are only two minor technical difficulties. First, recall that in this paper the $t$ stable points are defined in terms of the "true potential" $V(\cdot)$, and not in terms of the Brownian motion $W(\cdot)$ constructed by means of the KMT theorem (which was the approach of [1]). As in the derivation of (22), we can get round the lack of scaling properties of $V(\cdot)$ (which complicates $e . g$. controlling the number of $t^{1-\gamma_{t}^{z}}$-stable points between 0 and $z$ ) by using Lemma 2.3 .

The second difficulty is that, since $z$ can be of order $t^{a}$, the distance between two neighboring $t^{1-\gamma_{t}^{z}}$-stable points can be, in principle, rather large. This would complicate the things, because for the technique of [1] it is important that the distance between two neighboring $s$-stable points should be of order at most $\ln ^{M} s$, for a fixed $M$. To overcome this difficulty, simply note that, by Lemma 2.1 and Borel-Cantelli lemma, for any fixed $b>0$ we have that, $\mathbb{P}$-a.s., for large enough $t$ any distance between two neighboring $t^{b}$-stable points on the interval $[0, t]$ is not greater than $\ln ^{4} t$. 
To conclude, notice that Theorem 1.1 now follows from $(22,23)$, and the fact that $\mathrm{P}_{\omega}^{0}\left[\xi_{t} \geq z\right] \leq$ $\mathrm{P}_{\omega}^{0}\left[\max _{s \leq t} \xi_{s} \geq z\right]$.

\section{REFERENCES}

[1] F. Comets and S.Yu. Popov, Limit law for transition probabilities and moderate deviations for Sinai's random walk in random environment. Probab. Theory Relat. Fields 126 (2003) 571-609.

[2] A. Greven and F. den Hollander, Large deviations for a random walk in random environment. Ann. Probab. 22 (1994) $1381-1428$.

[3] Y. Hu and Z. Shi, The limits of Sinai's simple random walk in random environment. Ann. Probab. 26 (1998) 1477-1521.

[4] Y. Hu and Z. Shi, Moderate deviations for diffusions with Brownian potentials. (2003) Preprint PMA-792 available at www.proba.jussieu.fr/mathdoc/preprints/index.html\#2003

[5] B. Hughes, Random Walks and Random Environments. Vol. 2. Random Environments. The Clarendon Press, Oxford University Press, New York (1996).

[6] Z. Shi, Sinai's Walk via Stochastic Calculus, in Milieux Aléatoires, F. Comets and E. Pardoux Eds., Société Mathématique de France, Paris, Panoramas et Synthèses 12 (2001).

[7] A. Shiryaev, Probability. 2nd edn., Springer, New York (1989).

[8] Ya.G. Sinai, The limiting behavior of one-dimensional random walk in random medium. Theory Probab. Appl. 27 (1982) $256-268$.

[9] O. Zeitouni, Lecture Notes on Random Walks in Random Environment. (2003) Preliminary version at www-ee.technion.ac.il/ zeitouni/ps/notes1.ps 